Boise State University

ScholarWorks

Geosciences Faculty Publications and

Presentations

Department of Geosciences

2017

Uncertainty in a Geophysical Survey for a Bridge Foundation

Design

Paul Michaels

Boise State University

This is an author-produced, peer-reviewed version of this article. The final, definitive version of this document can be found online at GeoRisk 2017: Geotechnical Risk Assessment and Management, published by the American Society of Civil Engineers. Copyright restrictions may apply. doi: 10.1061/9780784480724.060 
This is an author-produced, peer-reviewed version of this article. The final, definitive version of this document can be found online at GeoRisk 2017: Geotechnical Risk Assessment and Management, published by the American Society of Civil Engineers. Copyright restrictions may apply. doi: $10.1061 / 9780784480724.060$

\title{
Uncertainty In A Geophysical Survey For A Bridge Foundation Design
}

\author{
Dr. Paul Michaels, P.E. M.ASCE ${ }^{1}$ \\ ${ }^{1}$ Center for Geophysical Investigation of the Shallow Subsurface, Boise State University, 1919 \\ University Drive, Boise, Idaho 83725-1535; email: pm@ cgiss.boisestate.edu
}

\begin{abstract}
In the preparation stage of a bridge replacement located in Boise Idaho, the Idaho Transportation Department (ITD) commissioned a geophysical survey to address uncertainties in the soil profile across the river. Differing site conditions can impact the final design and cost of a project. To address the geologic uncertainties under the planned bridge replacement, the refraction method was selected. Selection of a geophysical method bears its own risk. Refractions are used to map the top of the competent geologic formation below those soils incapable of supporting the structure. The refraction method requires that P-wave velocity increase at the boundary to be mapped. That requirement was not met in this project. Unexpected pore pressures in the formation to be mapped reduced the P-wave velocity by $30 \%$, causing the plan to fail. Fortunately, surface wave analysis provided an viable alternative to work with the data, but with less depth of penetration due to the lack of low frequency content in the air gun source.
\end{abstract}

\section{INTRODUCTION}

Knowledge of the soil and rock profile is a valuable aid in foundation design. Differing site conditions may affect choices such as H-piles, drilled shafts, or spread footings. Improved estimates of materials may be aided by a cross river survey of the soil profile. Geophysical methods can provide such a profile.

\section{Uncertainty in Choosing a Geophysical Method}

Each geophysical method depends on contrasts between the materials to be mapped and details in the soil profile. The refraction method requires an increase in velocity at the boundary to be mapped. Love waves require a wave guide. Rayleigh waves do not require a wave guide.

The refraction method is a geophysical approach often favored in mapping the depth to the top of geologic formations capable of supporting the planned structure. In a bridge replacement, the existing bridge allows deployment of either a seismic source or geophone from the bridge at stations spaced across the river. This is permitted by the reciprocity principle (Aki and Richards 1980). The noise conditions due to strong river flows makes deploying the source from the bridge, and placing the geophones on the shore the best configuration. Examples can be found in the literature (Michaels 2001a) and (Michaels 2004).

\section{THE REFRACTION METHOD}

The refraction of seismic waves follows the principle known as Snell's Law. This law relates the incident angle (measured from the normal to a boundary) to the refracted angle. If medium 1 is the incident side of the boundary, and medium 2 is the refracted side of the boundary, Snell's law is given as: 


$$
\frac{\sin \left(\theta_{1}\right)}{V 1}=\frac{\sin \left(\theta_{2}\right)}{V 2}
$$

where V1 and V2 are the respective velocities of the two media. When the velocity V2 is greater than V1, a critically refracted wave is possible and will travel along the boundary at the velocity V2, returning to the surface as shown in Figure 1A. Such rays, returning to the surface, result from rays incident at the critical angle. The critical angle is given by

$$
\theta_{1}=\sin ^{-1}\left(\frac{V 1}{V 2}\right)
$$

The velocities shown in Figure 1 are representative of those measured in the bore hole surveys. The case of Figure 1B matches the conditions at the project.
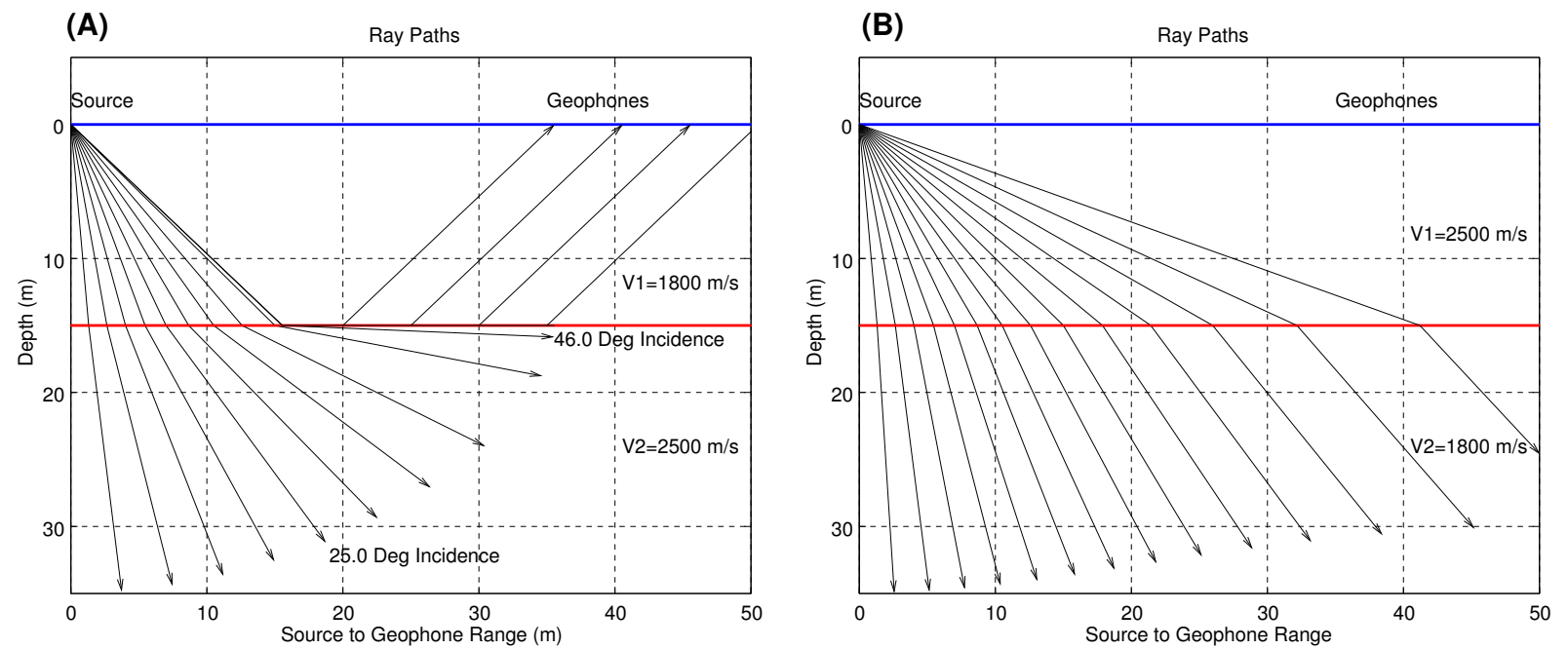

Figure 1: The refraction method requires a slower velocity in the soil above the boundary to be mapped. (A) Seismic waves return to the surface. (B) Waves will not return to the surface.

\section{FACTORS AFFECTING SEISMIC VELOCITIES}

Soils and rocks present seismic velocities which are affected by a number of factors. Important factors include:

1. Type of solid matrix.

2. Void ratio of the material.

3. Stress conditions present.

4. Fluids present in the pore spaces and degree of saturation.

Both compressional (P-wave) velocities and shear ( $\mathrm{S}$-wave) velocities directly relate to the elastic modulus of the geologic material. But laboratory and field studies have shown that these moduli are affected by void ratio and effective stress conditions (Hardin and Richart 1963), (EberhartPhillips et al. 1989), and (Wyllie et al. 1956). Further, empirical studies have related seismic velocities to geotechnical properties of interest. A relevant example would be bearing capacity 
(Tezcan and Ozdemir 2012), and (Kaptan 2012). The expectation is that large seismic velocities correlate with large bearing capacity. But if pore pressures rise, that reduces effective stress and lowers seismic velocities (Wyllie et al. 1958). This lowering of velocity may make velocity less representative of actual bearing capacity and strength.

\section{BORE HOLE GEOPHYSICS}

Eventually, the two bore holes were drilled and surveyed for both P- and SH-wave velocity. Table 1 gives details on the data acquisition. Bore hole HS-4 was located on the South bank of the Boise river, and HS-5 was located on the North bank. Coordinates and dimensions are to a local metric datum. The source was an inclined hammer as described in Michaels (1998). A surface reference 3-component Oyo geophone monitored any variations in the source waveform or trigger timing. The down-hole geophone was a BH-2 3-component sidewall clamping geophone (Crice 1996). Downhole geophone orientation was done by principle component analysis of horizontal component hodograms (Michaels 2001b).

Figures 2 and 3 show the down-hole vertical times measured from the surface hammer source to the 3-C geophone at depth. Elevations shown in feet are relative to sea level, the metric coordinate system is to the local origin (zero elevation approximately at ground level within the project). The inclined hammer source generates both $\mathrm{P}$ - and $\mathrm{SH}$-waves that are separated by signal processing methods. Each depth station receives two recordings of opposite source excitation polarity. The difference in recordings for that station enhances $\mathrm{SH}$-waves, the sum enhances P-waves. The slopes of the arrival time trends give the interval velocities for each formation. The negative velocity apparent in Figure 3A is an up-going wave reflected off the top of the $2721 \mathrm{~m} / \mathrm{s}$ formation and interferes with the down-going direct wave. The Glenn's Ferry formation was expected to be more supportive and exhibit a higher velocity than the soils at shallower depths. Thus, the original plan was to map a refraction off the top of the Glenn's Ferry formation. However, the velocity below this boundary decreases rather than increases. This condition makes refraction mapping impossible since the waves bend toward the normal on crossing the formation top and don't return to the surface (see Figure 1B).

TABLE 1: Down-hole Seismic Data Acquisition

\begin{tabular}{|l|r|r|}
\hline \hline \multicolumn{1}{|c}{ Setting } & \multicolumn{1}{c|}{ Bore Hole HS-4 } & \multicolumn{1}{c|}{ Bore Hole HS-5 } \\
\hline Number of samples & 4000 & 4000 \\
Sample Interval & $.00025 \mathrm{sec}$. & $.00025 \mathrm{sec}$ \\
Source Elevation & $+0.253 \mathrm{~m}$ & $+0.353 \mathrm{~m}$ \\
Subsurface Station Interval & $0.25 \mathrm{~m}$ & $.25 \mathrm{~m}$ \\
Bore Hole Coordinates (x,y) & $(-77.45,54.94) \mathrm{m}$ & $(-71.85,236.68) \mathrm{m}$ \\
Source Coordinates & $(-77.62,54.05) \mathrm{m}$ & $(-72.33,235.43) \mathrm{m}$ \\
Reference Phone Coordinates & $(-77.28,55.51) \mathrm{m}$ & $(-70.77,239.54) \mathrm{m}$ \\
Elevation Water Table & $-2.65 \mathrm{~m}$ & $-2.50 \mathrm{~m}$ \\
Date Surveyed & 02 July 2012 & 05 July 2012 \\
\hline \hline
\end{tabular}




\section{July 2012 Down-hole Data}

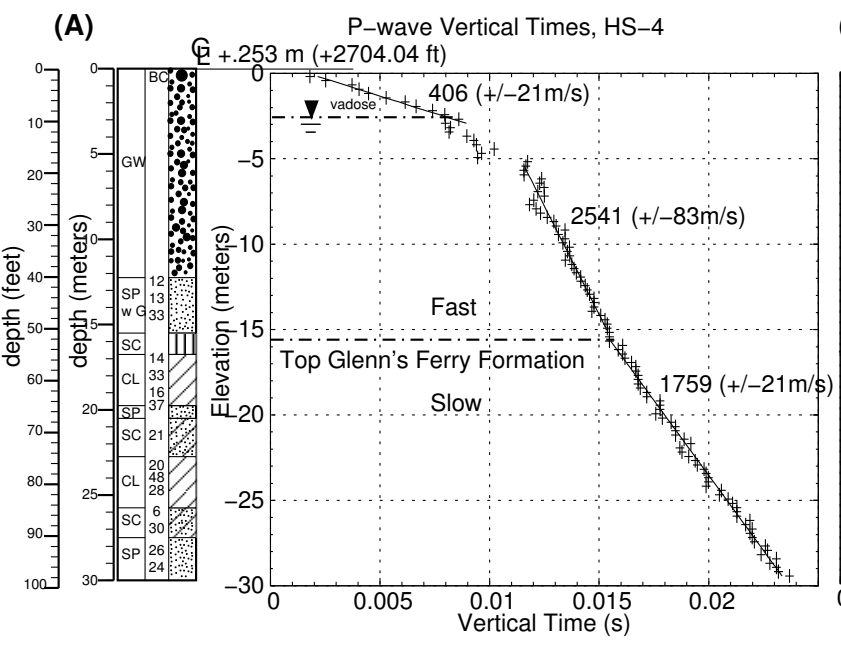

(B) S-wave Vertical Times, HS-4

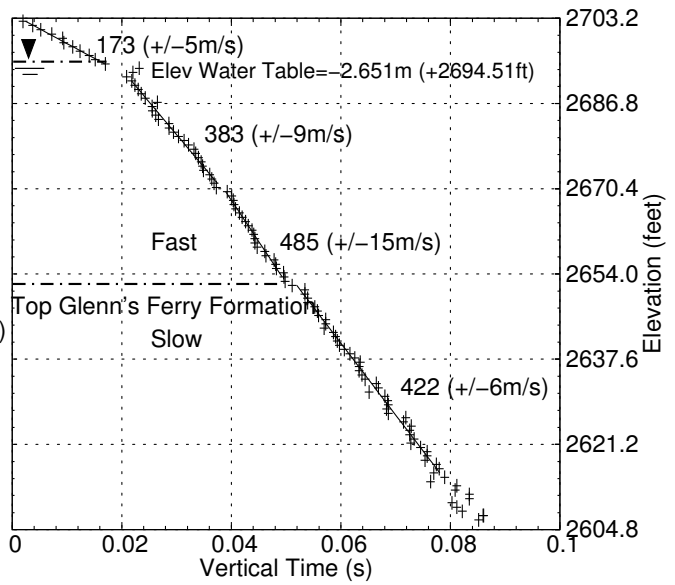

Figure 2: The unexpected $30 \%$ decrease in velocity at the top of the Glenns Ferry formation is likely due to elevated pore pressures. These conditions make mapping by refraction impossible.

(A)

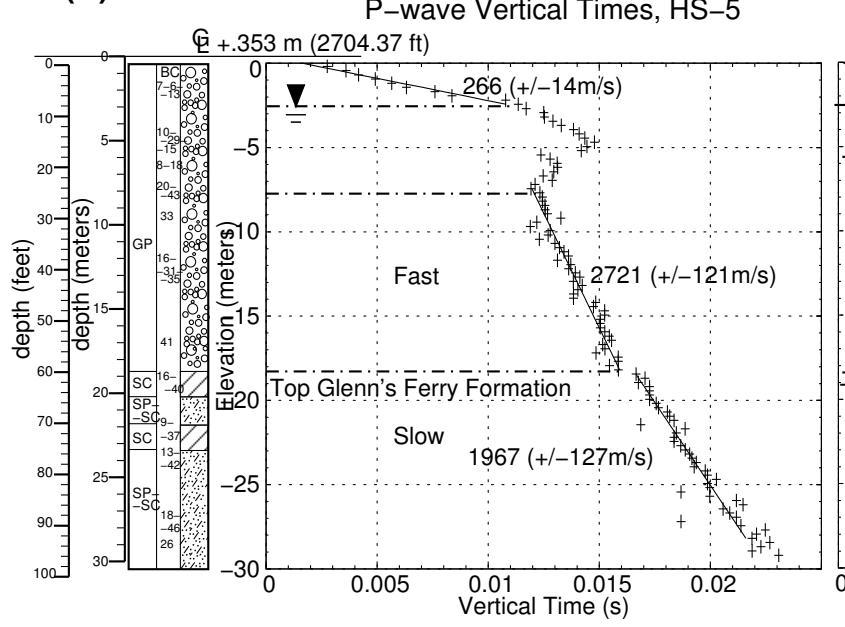

(B)

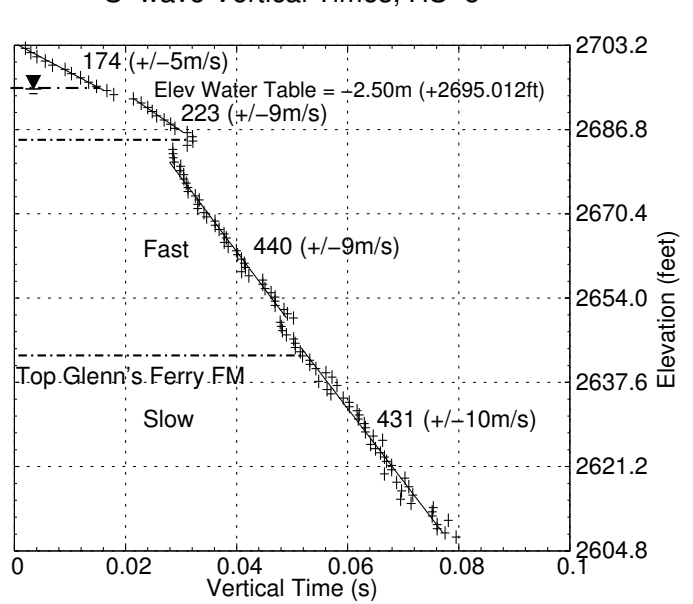

Figure 3: The data from the North bank bore hole also shows a decrease in velocity at the top of the Glenn's Ferry formation. An up-going reflection interferes with the down-going wave presenting a negative velocity in (A).

\section{AIR GUN IN RIVER SEISMIC RESULTS}

The cross river refraction survey was conducted before the bore holes were available. In that refraction survey an air gun was suspended from the existing bridge and fired near the water surface. Geophones were located along the shore and on an island, extending up stream from the bridge. Figure 4 shows base maps for the ends of the survey. Other examples of this type of design can be found in Michaels (2004). Details on the airgun design are given in Michaels (2001a). 
This is an author-produced, peer-reviewed version of this article. The final, definitive version of this document can be found online at GeoRisk 2017: Geotechnical Risk Assessment and Management, published by the American Society of Civil Engineers. Copyright restrictions may apply. doi: $10.1061 / 9780784480724.060$

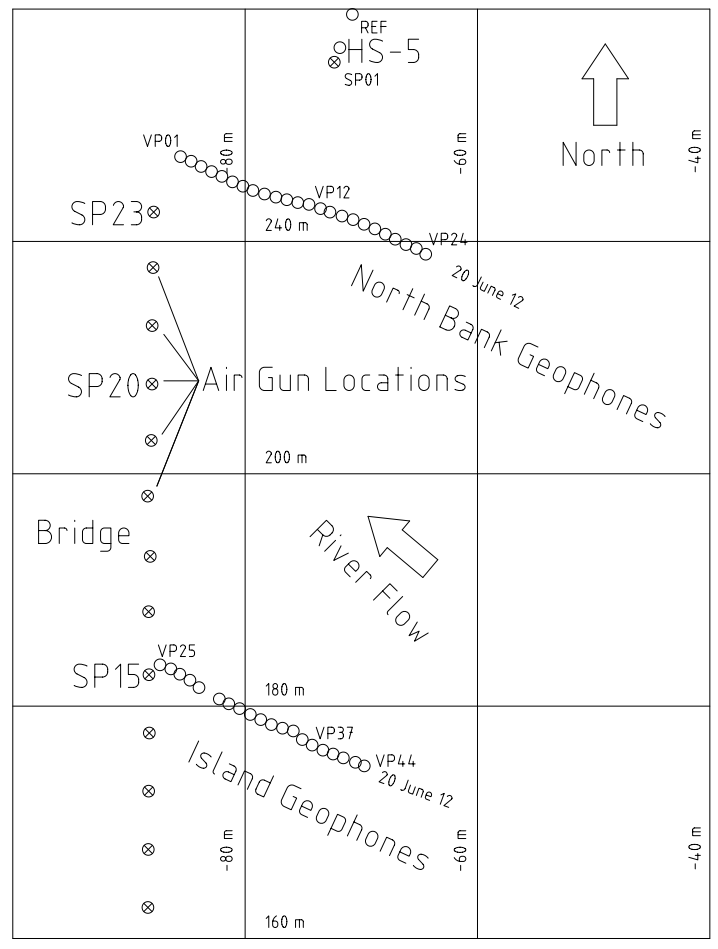

(a) North Bank

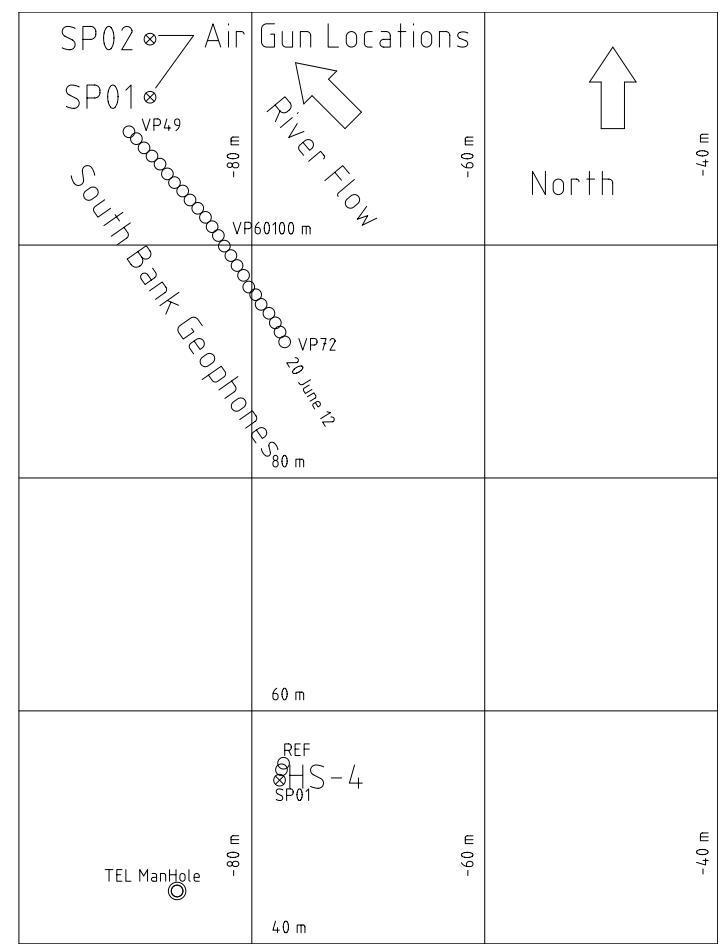

(b) South Bank

Figure 4: Maps showing locations of the boreholes, air gun stations (SP), and geophone locations (VP) on land.

\section{Observations}

The 20 June 2012 cross river air gun data can be examined from different points of view.

- Shot Gather. A single shot effort as seen on a collection of geophones.

- Receiver Gather. A single geophone's recorded signals from a collection of shots.

For example, consider the shot efforts along the existing bridge recorded on single geophone located on the island. The geophone signals at VP25 near shot SP15 are shown on Figure 5A. It is clear that refracted arrivals are absent, as we would expect as seen from the bore hole studies which were later conducted in July. The one apparent recorded signal is the direct air wave from the air gun to the geophone $(330 \mathrm{~m} / \mathrm{s}$ arrival velocity).

Next, we consider the shot gather for the air gun source at SP15, geophones trending South East from the bridge on the island (see Figure 4A). These recorded waves are shown in Figure 5B. The gap in the geophone spacing is due to a small stream on the island. Because of the island, SP15 required digging a shallow hole and filling it with water. The air gun was fired in the water filled hole to generate the signals. The fan shaped spreading of the waves away from the source is typical for a surface wave known as a Rayleigh wave (see Figure 5B). Rayleigh waves exhibit elliptical particle motion. The signals shown in Figure 5B are for the vertical component of motion and can be inverted for soil profile velocities. 


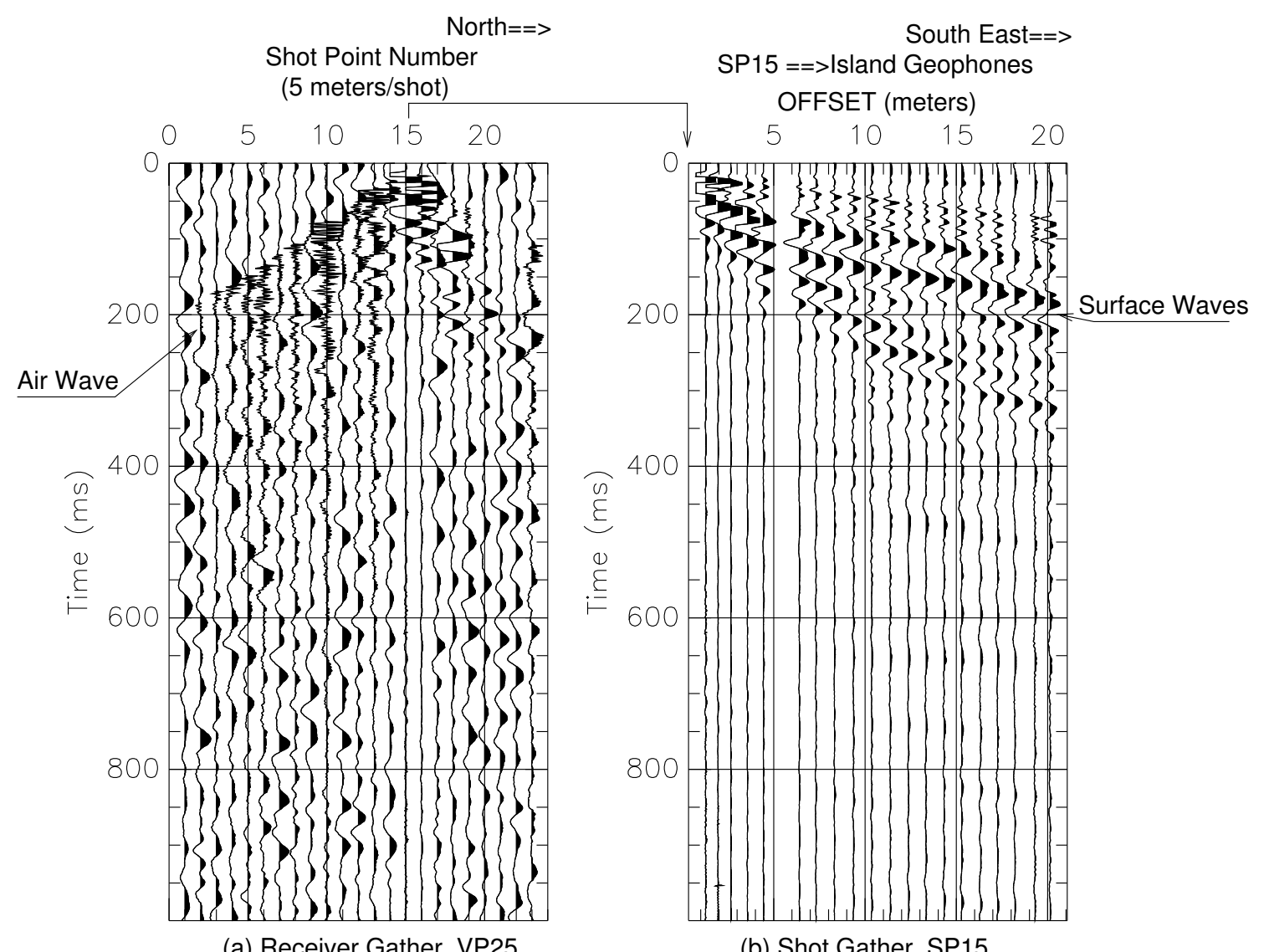

Figure 5: (A) Receiver gather showing signals from 23 air gun locations and (B) Shot gather showing SP15 signals recorded on island geophones (VP25-VP44). No refractions were recorded in (A), only an air wave. Surface waves were recorded on the island in (B).

\section{Surface Waves: Air Gun Source}

The air gun sourced surface wave data were inverted for soil profile S-wave velocities. A discussion on the inversion method may be found in Michaels (2011). Continuing with the sample from SP15 on the island, the data of Figure 5B were inverted to a S-wave velocity profile (see Figure 6B). We can observe that the air gun data are limited in low frequency content with increasing uncertainty in the signals below about $18 \mathrm{~Hz}$. This would not have been a problem if a refraction had been recorded. However, with surface waves, the deeper soil layers are probed by low frequency content. The increase in velocity at about -10 meters elevation is interpreted as the likely transition from slow to fast gravel (see Figure 6B). This transition is seen on Figure 3B at a shallower depth of about 5 meters in bore hole HS-5. It is unlikely that the deeper Glenn's Ferry formation was sensed due to the lack of low frequency content in the radiated surface waves.

\section{Surface Waves: Ambient Traffic Noise Source}

Early in the project (14 June 2012), a noise test was conducted to get a measure of the ambient noise generated by traffic on the bridge (Figure 7). Traffic vibrations are transfered from the bridge deck down to the soil through the foundations. Geophones were layed out to the South East from a 


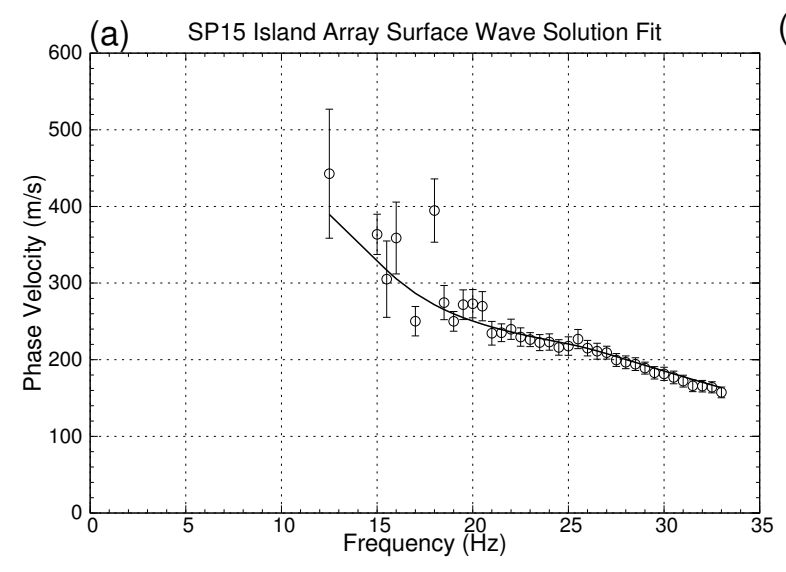

(b) SP15 Island Array Surface Wave Solution

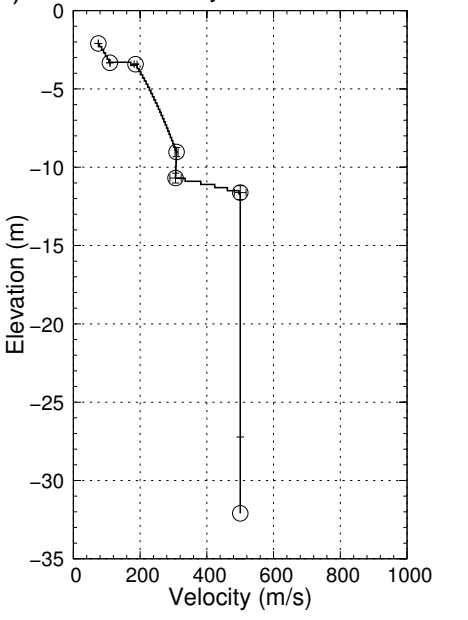

Figure 6: SP15 shot gather inversion of data in Figure 5B. Measured dispersion with $95 \%$ confidence limits in (A), and soil $S$-wave velocity profile (B). The solid curve in (A) is computed from solution (B).
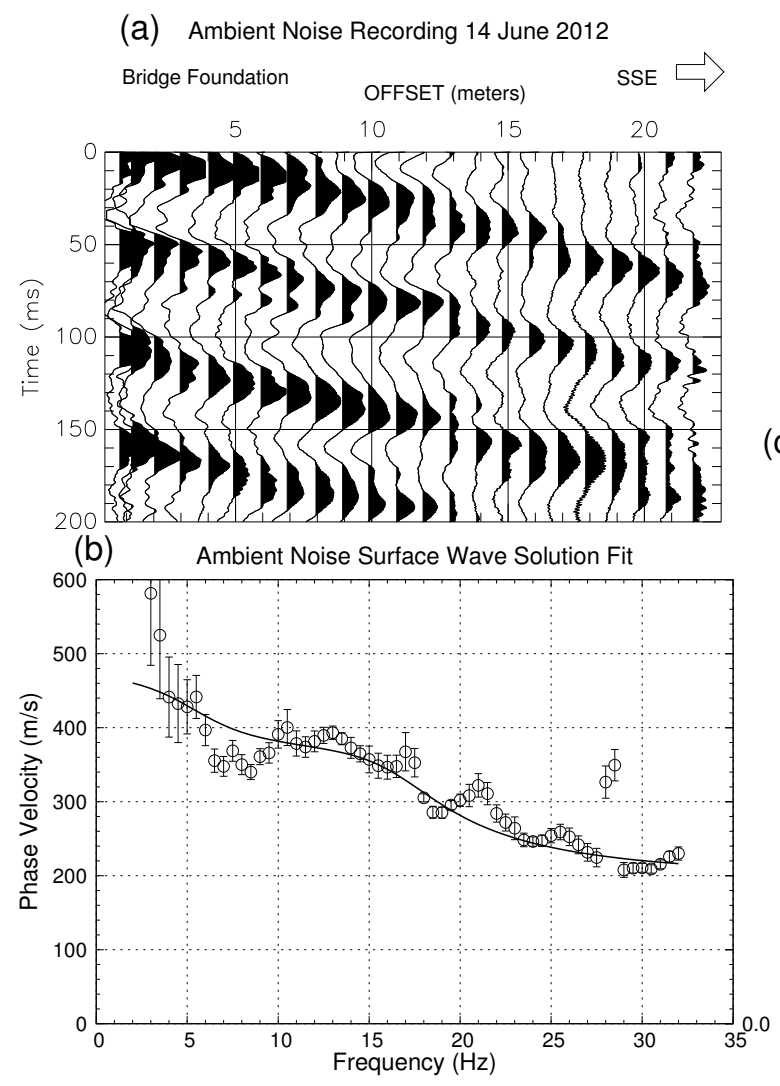

(c) Ambient Noise Surface Wave Solution

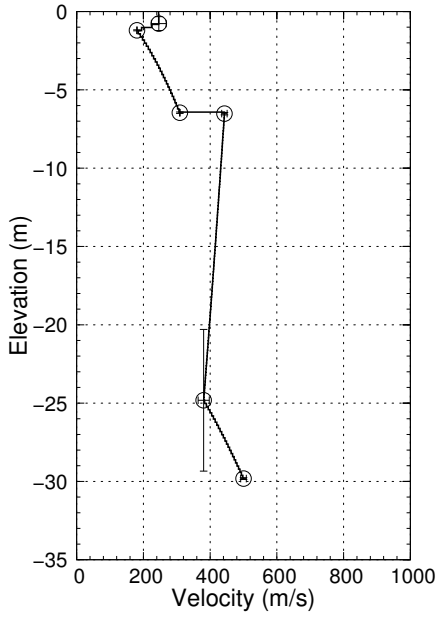

Figure 7: The first 200 msec of a 4 second recording of traffic noise (a), computed dispersion from the full 4.0 second record (b), and the solution for a subsurface soil profile which accounts for the dispersion. Compare traffic dispersion (b) with that in Figure 6 from an airgun. Note the lower frequency content here. 
foundation column. Characterizing the noise from traffic is helpful in the design of arrays formed to cancel that noise as an enhancement in a refraction survey. It is also possible to turn that noise recording into a signal since it senses the subsurface soil velocity profile.

Figure 7A shows the first 0.2 seconds of the 4.0 second recording of traffic noise. Also shown are the measured dispersion (B) and subsurface $S$-wave velocity profile that accounts for the measured dispersion. The solid curve in (B) is computed from the velocity profile in (C). The traffic noise provided the lower frequencies needed to probe deeper into the subsurface. These data agree well with the bore hole subsurface control and provide a boundary condition for the analysis of the air gun surface wave interpretations.

\section{Creating a Cross Section}

Creating a profile across the river was possible using the surface wave and bore-hole control. Figure 8 shows that the transition from slow to fast gravels could be mapped as S-wave velocities. This transition could not be mapped as a P-wave refraction because the P-wave velocities are dominated by the presence of water, where as the S-waves largely represent the frame. Another complication is that the bore-hole "P-wave" data are actually vertical component data which are likely contaminated by Rayleigh waves on the vertical component at very shallow depths. Consequently, the actual P-wave propagation velocities in the river are likely no less than $1500 \mathrm{~m} / \mathrm{s}$. It is likely that the transition from slow to fast gravels is only meaningful in the context of S-wave propagation.

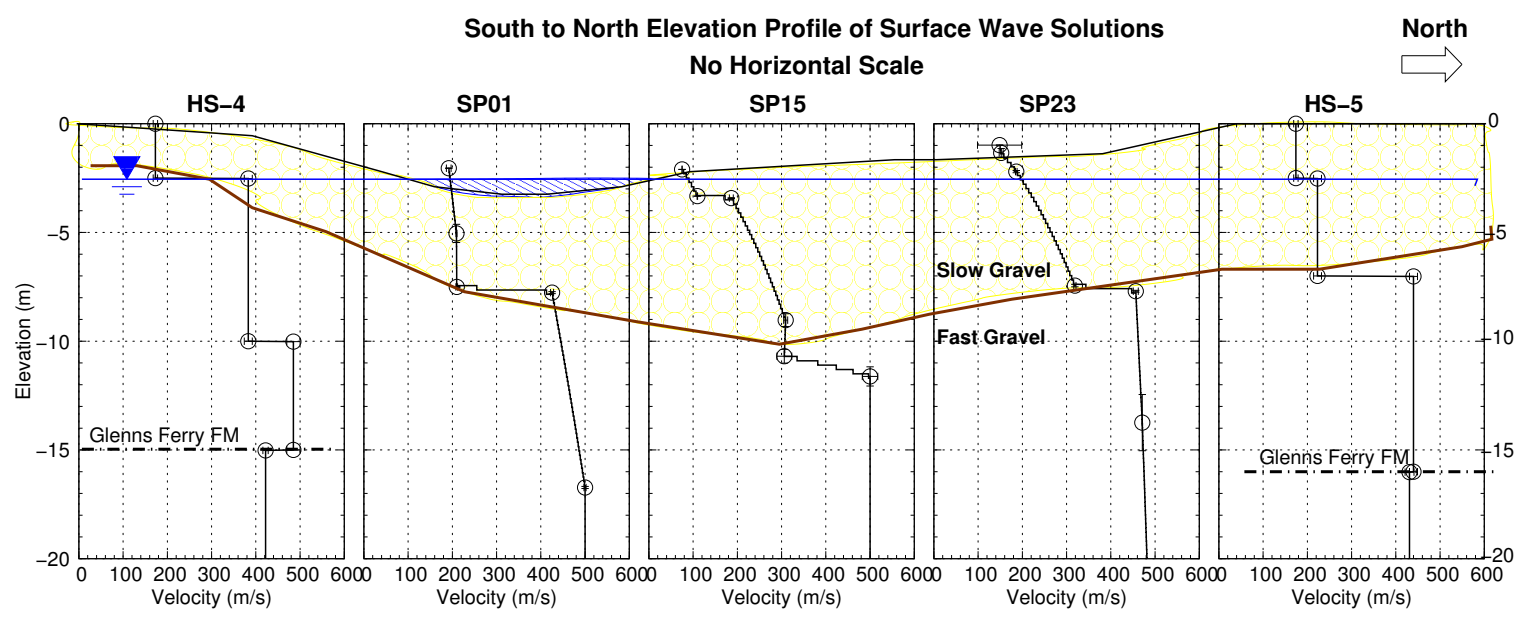

Figure 8: Shear wave velocities from down-hole and surface wave geophysics. The transition from slow to fast gravels is mapped, but the Glenn's Ferry formation could not be mapped.

\section{CONCLUSIONS}

The motivation for the collection of geophysical data was to avoid surprises due to differing site conditions. The foundation option favored in this setting is H-piles. Depth to bedrock or a soil layer with needed bearing capacity has often been determined by refraction geophysics. A geophysical survey will reduce the risk of surprises.

\section{Sources of Uncertainty and Risk}

Under favorable geologic conditions, the refraction method would be the method of choice. The recording of observed refracted arrival times are used to calculate the depth to competent soil 
This is an author-produced, peer-reviewed version of this article. The final, definitive version of this document can be found online at GeoRisk 2017: Geotechnical Risk Assessment and Management, published by the American Society of Civil Engineers. Copyright restrictions may apply. doi: $10.1061 / 9780784480724.060$

or rock formations capable of supporting the foundation. Uncertainty in that method resides in the interpretation of travel time which is a mix of velocity and distance. An increase in travel time may be due to a decrease in propagation velocity or an increase in distance to the structure being mapped. Michaels (1995) provides a discussion of those uncertainties.

However, in this project, the choice of the refraction method resulted in extreme uncertainty (the method failed all together due to the lack of a refractor). Factors that affect wave velocity are not singularly attributable to the soil/rock matrix and its bearing capacity. In particular, we saw that the stress conditions (excessive pore pressure) of the subsurface material decreased the velocity which prevented the return to the surface of the refractions needed to perform the calculations.

In this case, the geophysics was not a total failure. One may separate the data collection protocol from the method of analysis. A seismic source generates a number of wave fields. We were able to work with the surface waves despite the lack of refracted waves observable at the surface of the ground. Uncertainties exist in the surface wave method. In particular, the choice of seismic source determines the spectrum of radiated frequencies. The air gun fired from the bridge generates frequencies suitable for refraction analysis. These frequencies are above the band which would be most helpful in a surface wave analysis. Consequently, the surface wave analysis became limited in the depth of penetration. The air gun spectrum resided largely above $18 \mathrm{~Hz}$, and that limited the depth of penetration to about 10 meters maximum.

\section{Lessons Learned}

While it is tempting to specify an ASTM guideline for geophysical work, a contractual fixing of the geophysical method may introduce an additional risk. This occurs when the method is inappropriate for the geologic conditions. Had the geophysics been restricted to refraction analysis only, the geophysics would have been of little use. We were fortunate in this case that no ASTM guideline was imposed.

The recording of traffic noise test turned out to be useful as an alternative seismic source to the air gun. Had we recorded more traffic noise from the bridge, we would have been able to explore deeper into the subsurface because of the richer low frequency content of traffic noise.

Finally, scheduling of activities can affect the ultimate uncertainties in a project. The bore hole drilling was delayed before it would have been of most use. Conducting the down-hole surveys would have alerted us to the problem with a refraction survey. Further, the first attempt at drilling borehole HS-4 had to be abandoned due to the elevated pore pressures. A different rig was brought in to complete the boreholes adding further delays. The schedule for the cross river refraction attempt was driven by the need to complete that work before the rafting season started. It is likely that the refraction method would have then been abandoned in favor of a surface wave alternative. Rather than collecting air gun data during breaks in traffic, we might have used the traffic itself as a source. 


\section{REFERENCES}

Aki, K. and Richards, P. (1980). Quantitative Seismology. W. H. Freeman and Co. 557 p.

Crice, D. (1996). BHG-2, BHG-3 borehole geophone operation manual. GeoStuff, 19623 via Escuela Dr., Saratoga, Ca 95070 16p.

Eberhart-Phillips, D., Han, D. H., and Zoback, M. D. (1989). "Empirical relationships among seismic velocity, effective pressure, porosity, and clay content in sandstone." Geophysics, 54(1), 82-89.

Hardin, B. O. and Richart, F. E. (1963). "Elastic wave velocities in granular soils." J. Soil Mech. Found. Div. ASCE, 89(SM1), 33-65.

Kaptan, K. (2012). "A refined formula for the allowable soil pressure using shear wave velocities." Journal of Civil Engineering and Costruction Technology, 3(3), 90-98.

Michaels, P. (1995). "A geophysical site investigation for a bridge foundation in a narrow canyon." Environmental \& Engineering Geoscience, 1(2), 219-226.

Michaels, P. (1998). "In situ determination of soil stiffness and damping." Journal of Geotechnical and Geoenvironmental Engineering, 124(8), 709-719.

Michaels, P. (2001a). "Use of engineering geophysics to investigate a site for a bridge foundation." in Foundations and Ground Improvement, T.L. Brandon, editor, GSP 113, ASCE, 715-727.

Michaels, P. (2001b). "Use of principal component analysis to determine down-hole tool orientation and enhance SH-waves." Journal of Environmental and Engineering Geophysics, 6(4), $175-183$.

Michaels, P. (2004). "Design of geophysical surveys in transportation.” Geotechnical Engineering for Transportation Projects GSP 126, Vol. 2, ASCE, 1832-1839. M. K. Yegian and E. Kavazanjian editors.

Michaels, P. (2011). "Establishing confidence in surface wave determined soil profiles." GeoRisk 2011 Geotechnical Risk Assessment and Management, C. H. Juang, K. K. Phoon, A. J. Puppala, R. A. Green, and G. A. Fenton, eds., number 837 in GSP 224, GeoIstitute of ASCE.

Tezcan, S. S. and Ozdemir, Z. (2012). "Allowable bearing pressure in soils and rocks through seismic wave velocities." Earth Science Research, 1(1), 98-108.

Wyllie, M. R. J., Gregory, A. R., and Gardner, G. H. F. (1958). "An experimental investigation of factors affecting elastic wave velocities in porous media.” Geophysics, XXIII(3), 459-493.

Wyllie, M. R. J., Gregory, A. R., and Gardner, L. W. (1956). "Elastic wave velocities in heterogeneous and porous media." Geophysics, XXI(1), 41-70. 